\title{
Arcanobacterium pyogenes and encrusted pyelitis
}

Albert Semaan*,1, Georges Abi Tayeh'1, Josselin Abi Chebel ${ }^{1}$, Rabih Hallit ${ }^{2,3}$, Matta Matta ${ }^{3,4}$ \& Pascal Hajj ${ }^{5}$

${ }^{1}$ Urology Department, Faculty of Medicine, Saint-Joseph University, Beirut, Lebanon

${ }^{2}$ Faculty of Medicine, Holy Spirit University of Kaslik - USEK, Lebanon

${ }^{3}$ Infectious Disease Department, BelleVue Medical Center, Mansourieh, Lebanon

${ }^{4}$ Faculty of Medicine, Saint-Joseph University, Beirut, Lebanon

${ }^{5}$ Urology Department, BelleVue Medical Center, Mansourieh, Lebanon

*Author for correspondence: Tel.: +961961 7052 4911; albertsemaan9180@gmail.com

\begin{abstract}
Aim: Trueperella pyogenes is known to affect cattle, but was never isolated as a cause of human urinary tract infections. Clinical case: A 69-year-old male presented for recurring low urinary tract symptoms after a 20-day ciprofloxacin regimen for prostatitis. He previously underwent open right nephrolithotomy and left ureterovesical junction reimplantation for an iatrogenic distal ureteral stricture. Computed tomography showed spontaneous cortical calcifications; renoscopy was performed and deep cultures from the pelvis were taken; culture on chocolate agar revealed T. pyogenes. Intravenous teicoplanin for 3 weeks resulted in resolution of low urinary tract symptoms with regression of bladder and ureteral thickening. Conclusion: T. pyogenes can cause encrusted pyelitis in humans especially evoked in a context of persisting or recurring urinary tract infections.
\end{abstract}

Lay abstract: We present a case report of a 69-year-old male presenting with persisting low urinary tract symptoms after a ciprofloxacin regimen received for prostatitis. Diagnostic work-up revealed encrusted pyelitis based on the demonstration of spontaneous renal cortical calcifications on imaging, and growth of the bacteria Trueperella pyogenes on chocolate agar. Treatment with intravenous teicoplanin given for 3 weeks resulted in permanent resolution of low urinary tract symptoms.

First draft submitted: 7 February 2019; Accepted for publication: 26 September 2019; Published online: 9 December 2019

Keywords: Arcanobacterium pyogenes $\bullet$ atypical pathogens $\bullet$ encrusted pyelitis $\bullet$ human $\bullet$ pyelitis $\bullet$ renal calcifications • Trueperella pyogenes • urinary tract infections

\section{Case presentation}

We herein report the case of a 69-year-old male with a past medical history of hypertension, dyslipidemia and a remote history of smoking (stopped 15 years ago), who works as a car painter and has no direct contact with animals, consulting for recurring lower urinary tract symptoms (LUTS) after completing a 20-day course of ciprofloxacin for prostatitis. His past urologic history included urolithiasis for which he underwent right open nephrolithotomy, left ureterovesical junction reimplantation for most likely iatrogenic distal ureteral stricture, benign prostatic hyperplasia treated with tamsulosin and recurring macrohematuria with sterile urine cultures. He also had an episode of acute prostatitis 1-year prior, treated by a 20-day course of ciprofloxacin with persistence of LUTS and sterile pyuria. His current medication regimen consists of aspirin, atorvastatin, vitamin D, perindopril amlodipine and tamsulosin.

A written informed consent was obtained from the patient for publication of his case report and any accompanied images. A copy of the written consent is available for review. The patient signed the written informed consent in his treating physician's clinic.

Sperm culture showed no bacterial growth. Abdomen and pelvis computed tomography (CT) scan performed before injection of intravenous (IV) contrast showed a multiscarred left kidney with spontaneously hyperdense cortical images, likely in keeping with cortical calcifications, associated to multiple cortical cysts, some of them spontaneously hyperdense. A tomographic complement after IV contrast injection with acquisitions obtained at 


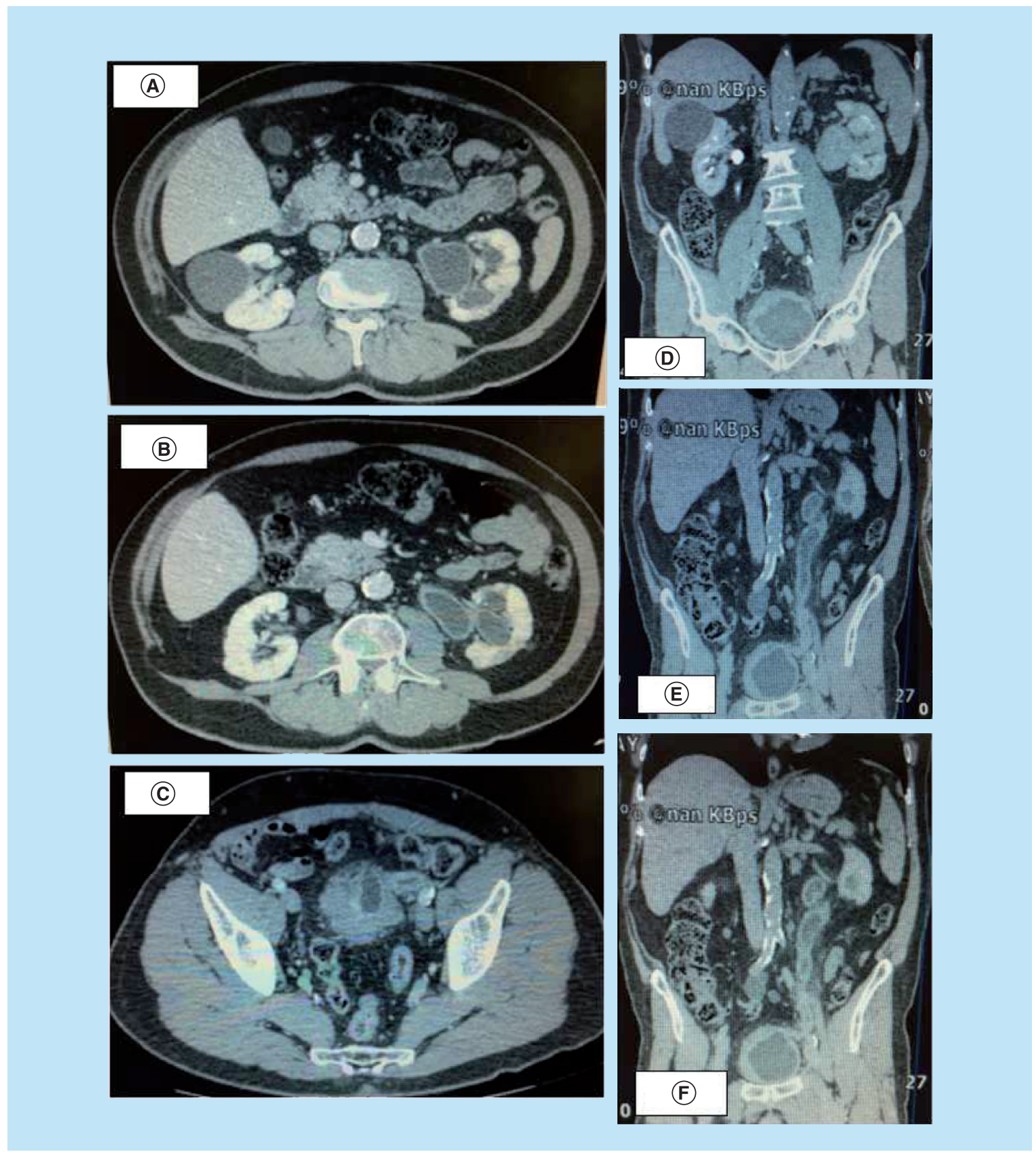

Figure 1. (A-C) Abdominopelvic computed tomography scan before treatment with teicoplanin showing calcification and urothelial wall thickening. (D-F) Abdominopelvic computed tomography scan before treatment with teicoplanin showing calcification and urothelial wall thickening.

portal and delayed phases showed a multiscarred left kidney with a dilated tortuous hypotonic ureter and a thickened wall showing significant enhancement with surrounding fat streaking, multiple nonenhancing filling defects in the left renal pelvis (Figure 3), and satellite lymph nodes measuring up to $12 \mathrm{~mm}$ of lesser diameter. A thickened multidiverticular bladder wall, suggestive of bladder and upper tract urothelial tumor (Figure 1) was also noted. Urine $\mathrm{pH}$ was 6.5.

A cystoscopy with ureteroscopy and left ureteral and pelvic biopsies were performed showing a follicular cystitis, inflammatory ureteral mucosa, granulation tissue, ectatic lymph vessels, congestive and edematous changes, with no signs of malignancy. Renal pelvis showed no papillary tumor but cloudy, grayish urine with floating whitish debris instead. Those findings were suggestive of atypical infections. Deep cultures from the pelvis were taken 
showing no bacterial growth of usual pathogens. Therefore, work-up for atypical infections was initiated. PCR for tuberculosis was negative but specific culture on media for corynebaterial infections, chocolate agar per se, showed a growth of Trueperella pyogenes, formerly known as Arcanobacterium pyogenes. Pathogen identification was done by biochemical testing through an analytical profile index. Trypticase soy agar/broth with defibrinated sheep blood was the special media used for culture. The patient was treated with IV teicoplanin for 3 weeks. Antibiotic regimen included a bolus loading dose then a maintenance perfusion dose given over $30 \mathrm{~min}$. Antibiotic treatment resulted in resolution of his urinary symptoms. Teicoplanin was used based on susceptibility tests performed in vitro on the isolated strain. Teicoplanin, along with vancomycin, has been proven to be the antibiotic to which all isolates of corynebacterial species were susceptible [1]. This is the main reason behind the choice of teicoplanin for management of the patient.

Patient management was performed on regular floor by a multidisciplinary team composed of urology and infectious diseases specialists. The treatment strategy was chosen based on clinical criteria since the patient demonstrated hemodynamic stability since his initial presentation and during his whole hospital stay. Response to treatment was mainly evaluated clinically, by resolution of low urinary tract symptoms.

Five months later, the patient was still LUTS-free; an abdomen and pelvic CT scan was performed, showing a significant decrease in the left ureter and bladder wall thickening and surrounding fat streaking (Figure 2).

\section{Background}

To our knowledge, this is the first case report that identifies T. pyogenes/A. pyogenes as the cause of encrusted pyelitis in humans. It was shown to be the relevant pathogen since the patient failed many empiric antibiotic regimens but fully recovered only after appropriate antibiotic therapy with teicoplanin, and no other pathogen was isolated from the patient's pelvis when culture was performed over regular and specific media.

Encrusted pyelitis was initially described in 1992 [2]. It is a rare chronic infectious and inflammatory condition of the renal pelvis characterized by calcific deposits or encrustation of the urothelium typically by phosphate and ammonium-magnesium salts [3,4]. It is a threatening disorder that can destroy the renal graft of transplanted patients but it can also affect native kidneys [5]. It has been described in immunocompromised or debilitated patients who have undergone urologic procedures to treat a variety of inflammatory or neoplastic conditions and patients treated with long-term broad-spectrum antibiotics. Reported cases are scant, even among renal transplant recipients who are at increased risk. Diagnosed patients are rare, with a reported incidence of $0.2 \%$ [6]. Alkaline-encrusted pyelitis is an infectious disease characterized by encrustations in the wall of the upper urinary tract, surrounded by severe inflammation [2]. For this reason, most consider it a healthcare-associated disease [7]. It is well-known in adults but rarely identified in children despite many case reports [8].

The disease is caused by an urea-splitting bacteria, most often Corynebacterium urealyticum, a Gram-positive, slow-growing microorganism that is multiresistant against antibiotics [2]. It has also caused one case of encrusted prostatitis [9], as well as severe fatal septic shock in immunocompetent patients [10,11]. It has even been responsible for nephrolithiasis associated with C. urealyticum urinary tract infection (UTI) in a dog [12]

Missing the diagnosis is not unusual since this germ has a lag phase higher than the regular conservation of urine culture (more than $48 \mathrm{~h}$ ) and needs special culture media to grow in; therefore, it must be specified for the microbiologist. There is often a delay between the onset of symptoms and the diagnosis, which is usually more than 1 month duration in all cases [8].

The prevalence of infection by $C$. urealyticum and obstructive uropathy is increasing [13]. Clinical manifestations of encrusted pyelitis are nonspecific, including gross hematuria and pyuria, eventually with elimination of pus, urinary debris and stones [14]. Main complications of encrusted pyelitis include obstructive uropathy associated with end-stage renal failure, renal abscesses, ureteral stenosis and renal graft nephrectomy [15].

Imaging is essential for diagnosis. Thoumas et al. reported that the abdomen/pelvis CT scan without contrast is the baseline examination [16]. Calcifications covering the urothelium encrusting the wall of renal calyces, the renal pelvis, the ureter and the bladder must be distinguished from staghorn calculi $[15,16]$. CT scan is the modality of choice for the diagnosis and follow-up of the calcifications after treatment [17].

Corynebacterium species are always sensitive to vancomycin and teicoplanin [18]. Some authors recommend conservative management with antibiotics and oral dissolution of the plaques using urine acidifying agents such as ammonium chloride in capsule [19]. Percutaneous nephrostomy tubes allowing irrigation with Thomas' acid solution are possible, the outflow being ensured by ureteric catheters [20]. Long-term follow-up is also necessary [21]. 

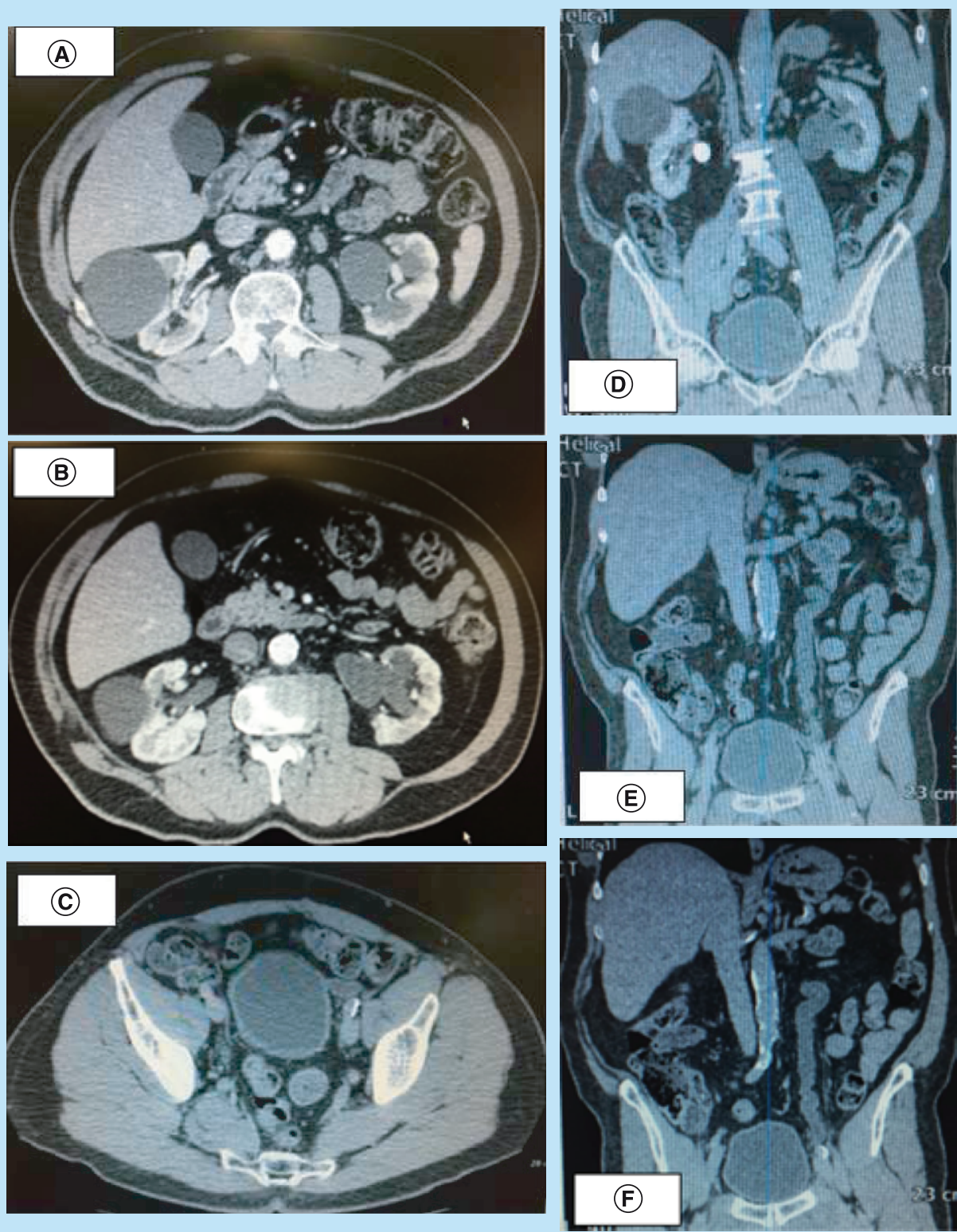

Figure 2. (A-C) Abdominopelvic computed tomography scan after treatment with teicoplanin showing complete regression of urothelial calcification and mucosal thickening. (D-F) Abdominopelvic computed tomography scan after treatment with teicoplanin showing complete regression of urothelial calcification and mucosal thickening.

Arcanobacterium pyogenes aka T. pyogenes is a Gram-positive, pleomorphic bacillus, a commensal normal inhabitant of the mucous membranes of domestic animals, such as cattle, sheep, swine and goats and an opportunistic pathogen of economically important livestock, causing diseases as diverse as mastitis, liver abscesses and pneumonia [22,23]. It has not been isolated as part of the normal human flora [22] and is rarely a cause of infection in humans, with infections mostly related to living in rural areas and contact with animals [24]. There are three published reports of human A. pyogenes endocarditis in the literature [25], a case of sepsis in a farmer in Brazil [23] and three cases of wound infections associated with $A$. pyogenes, reported for the first time in India in patients with diabetes and a past history of Hansen's disease, residing in a rural area in close contact with animals [26]. A. pyogenes is usually susceptible to benzyl penicillin, ampicillin, gentamicin and macrolides and resistant to cotrimoxazole, streptomycin and tetracyclines [27]. 
Figure 3. (A-C) Abdominal computed tomography scan showing multiple nonenhancing filling defects on the left renal pelvis at delayed phase before treatment with teicoplanin.

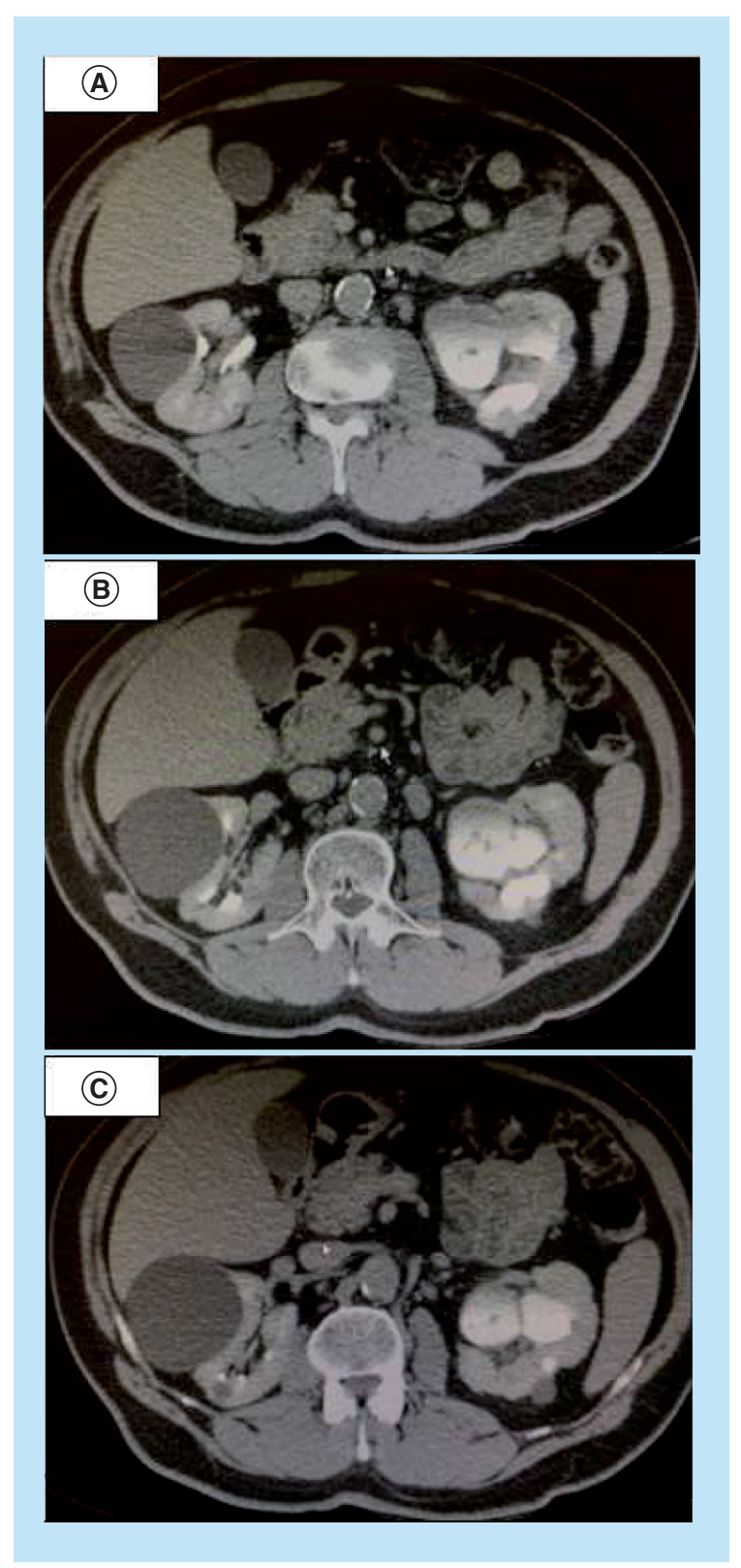

\section{Discussion}

In the presented case, the persistence of severe LUTS despite prolonged previous antibiotic regimens raised suspicion of the likelihood of a multiresistant pathogen. In addition, CT scan findings of renal cortical calcifications and debris found on cystoscopy/ureteroscopy pushed the multidisciplinary team to further the investigations to isolate an uncommon pathogen incriminated in the sustained and recurrent UTIs. Although some findings were against encrusted pyelitis such as the urine $\mathrm{pH}$ of 6.5 (nonalkaline), the presence of debris in the patient's pelvis and bladder in addition to cortical renal calcifications strongly pointed to the diagnosis, especially after the patient only improved after a teicoplanin regimen having failed many previous antibiotic therapies. Furthermore, it has to be kept in mind that the patient previously underwent an ureterovescial junction reimplantation, making it an additional risk factor of contracting encrusted pelitits. The Arcanobacterium species identified in the patient's culture was sensitive to penicillin, rifampicin, gentamycin, clindamycin vancomycin and resistant to sulfamethoxazole-trimethoprim, which is consistent with the known microbiology features of these bacteria. Pathogen sensitivity was tested through a susceptibility test performed in vitro, in a microbiology laboratory setting. Teicoplanin was the first and only antibiotic that was used to treat this patient. 


\section{Conclusion \& future perspective}

In conclusion, the current report highlights the importance for urologists and infectious disease specialists to be alert to the possible diagnosis of encrusted pyelitis. In fact, despite encrusted pyelitis being a rare UTI, physicians should evoke this entity in the context of a UTI that is resistant to common treatment regimens or when no bacteria are found in cultures. It also strongly suggests that $A$. pyogenes aka $T$. pyogenes can be pathogenic to humans and should be considered by microbiologists for diagnostic and therapeutic purposes whenever a UTI with negative culture and atypical symptoms is present.

\section{Executive summary}

Case presentation

- A 69-year-old male presented with recurring lower urinary tract symptoms despite a complete course of antibiotic for prostatitis.

- Right open nephrolithotomy, left ureterovesical junction reimplantation, benign prostatic hyperplasia treated with tamsulosin are his past urologic history.

- Recurring macrohematuria and lower urinary tract symptoms with sterile urine cultures are his chief complaints.

- Abdomen and pelvis computed tomography (CT) scan showing multiscarred left kidney, renal pelvis calcification and thickening of his urothelial bed are suggestive of atypical infection or tumor.

- Renal pelvis cultures on special media showed Trueperella pyogenes growth.

- Intravenous Teicoplanin for 3 weeks resulted in resolution of his clinical symptoms and radiological features.

Background

- This is the first case report identifying $T$. pyogenes as the cause of encrusted pyelitis in humans.

- Encrusted pyelitis is usually caused by corynebacterial infection.

- Encrusted pyelitis results in alkaline urine $\mathrm{pH}$, calcific deposits of the by phosphate and ammonium-magnesium salts.

- Diagnosis of encrusted pyelitis is usually missed and needs special media cultures.

- Abdomen/pelvis CT scan without contrast is the baseline examination.

- Corynebacterium species are always sensitive to vancomycin and teicoplanin.

- Arcanobacterium pyogenes, renamed T. pyogenes, is a Gram-positive, pleomorphic bacillus, a commensal normal inhabitant of the mucous membranes of domestic animals rarely pathogenic in humans.

Discussion

- Presence of debris in the patient's pelvis and bladder in addition to cortical renal calcifications strongly pinpoint to the diagnosis of encrusted pyelitits (EP).

- Previous urologic surgeries are a risk factor for EP.

- Teicoplanin is the treatment of choice for EP.

Conclusion \& future perspective

- It is important for urologists and infectious disease specialists to be alert to the possible diagnosis of encrusted pyelitis.

- EP should be evoked in the context of a urinary tract infection that is resistant to common treatment regimens or when no bacteria are found in cultures.

- Arcanobacterium pyogenes aka T. pyogenes can be pathogenic to humans and cause urinary tract infection.

Author contributions

A Semaan contributed to the conception of the case report, data collection and drafting and critical revision of the article. GA Tayeh contributed to collection and assembly of data and revision of the article. JA Chebel made contributions in drafting the article. R Hallit and M Matta contributed to data collection and revision of the article. P Hajj contributed to conception of the case report and final approval of the article.

Financial \& competing interests disclosure

The authors have no relevant affiliations or financial involvement with any organization or entity with a financial interest in or financial conflict with the subject matter or materials discussed in the manuscript. This includes employment, consultancies, honoraria, stock ownership or options, expert testimony, grants or patents received or pending, or royalties.

No writing assistance was utilized in the production of this manuscript.

Ethical conduct of research

The authors state that they have obtained appropriate institutional review board approval or have followed the principles outlined in the Declaration of Helsinki for all human or animal experimental investigations. The authors state that they have obtained verbal and written informed consent from the patient/patients for the inclusion of their medical and treatment history within this case report. 


\section{Open access}

This work is licensed under the Creative Commons Attribution 4.0 License. To view a copy of this license, visit http://creativecommons.org/licenses/by/4.0/

\section{References}

Papers of special note have been highlighted as: $\bullet$ of interest; $\bullet \bullet$ of considerable interest

1. Olender A. Antibiotic resistance and detection of the most common mechanism of resistance (MLSB) of opportunistic corynebacterium. Chemotherapy 59(4), 294-306 (2013).

- Shows the crucial role of teicoplanin in corynebacterial infections.

2. Morales JM, Aguado JM, Diaz-Gonzalez R et al. Alkaline-encrusted pyelitis/cystitis and urinary tract infection due to corynebacterium urealyticum: a new severe complication after renal transplantation. Transplant. Proc. 24(1), 81-82 (1992).

3. van Hooland S, Vandooren AK, Lerut E, Oyen R, Maes B. Alkaline encrusted pyelitis. Acta Clin. Belg. 60(6), 369-372 (2005).

4. Pierciaccante A, Pompeo ME, Fabi F, Venditti M. Successful treatment of Corynebacterium urealyticum encrusted cystitis: a case report and literature review. Infez. Med. 15(1), 56-58 (2007).

5. Hertig A, Duvic C, Chretien Y, Jungers P, Grünfeld J-P, Rieu P. Encrusted pyelitis of native kidneys. J. Am. Soc. Nephrol. 11(6), 1138-1140 (2000).

6. Devine PA, Courtney AE. Alkaline-encrusted pyelitis in a renal allograft. Kidney Int. Rep. 4(1), 174-177 (2018).

7. Meria P, Desgrippes A, Arfi C, Le Duc A. Encrusted cystitis and pyelitis. J. Urol. 160(1), 3-9 (1998).

8. Meria P, Margaryan M, Haddad E, Dore B, Lottmann HB. Encrusted cystitis and pyelitis in children: an unusual condition with potentially severe consequences. Urology 64(3), 569-573 (2004).

9. Serrano-Brambila E, López-Sámano V, Montoya-Martínez G, Holguín-Rodríguez F, Maldonado-Alcaraz E. Encrusted prostatitis: case report and literature review. Actas Urol. Esp. 30(3), 321-323 (2006).

10. Audard V, Garrouste-Orgeas M, Misset B et al. Fatal septic shock caused by Corynebacterium D2. Intensive Care Med. 29(8), 1376-1379 (2003).

11. Moreno Arcas P, Anglada Curado FJ, Prieto Castro R et al. Encrusted pyelitis. Lithiasic disease with infectious etiology. Actas Urol. Esp. 26(1), 53-56 (2002).

12. Fleischhacker S, Horstmann C, Hartmann K, Schubert S, Dorsch R. Carbonate apatite nephrolithiasis associated with Corynebacterium urealyticum urinary tract infection in a dog. Aust. Vet. J. 92(5), 161-165 (2014).

13. Sánchez-Martín FM, López-Martínez JM, Kanashiro-Azabache A et al. Corinebacterium urealyticum: increased incidence of infection and encrusted uropathy. Actas Urol. Esp. 40(2), 102-107 (2016).

14. Alkaline-encrusted pyelitis and cystitis: an easily missed and life-threatening urinary infection. Semantic Scholar (2011). https://www.ncbi.nlm.nih.gov/pmc/articles/PMC3070375/

- Shows the clinical manifestation and diagnostic criteria of incrusted pyelitis and how serious this infection is.

15. Saljoghi R, Lipsker A, Caillet K et al. Encrusted uretero-pyelitis: case report. Urol. Case Rep. 7, 58-60 (2016).

16. Thoumas D, Darmallaicq C, Pfister C et al. Imaging characteristics of alkaline-encrusted cystitis and pyelitis. AJR Am. J. Roentgenol. 178(2), 389-392 (2002).

-. Shows the radiological criteria for encrusted pyelitis that helped us with our diagnosis.

17. Vázquez V, Morales MD, Serrano C, Reus M, Llorente S, García J. Corynebacterium urealyticum in renal trasplantation. CT and sonography imaging characteristics of encrusted cistitis and pielitis. Nefrologia 24(3), 288-293 (2004).

18. Meria P, Jungers P. Encrusted pyelitis: an underdiagnosed condition? Nephrol. Dial. Transplant. 15(7), 943-945 (2000).

19. Khallouk A, Wallerand H, Kleinclauss F, Bittard H, Bernardini S. Conservative management of corynebacterium urealyticum encrusted cystitis. Prog. Urol. 16(4), 496-498 (2006).

20. Meria P, Desgrippes A, Fournier R et al. The conservative management of corynebacterium group D2 encrusted pyelitis. BJU Int. 84(3), 270-275 (1999).

21. Meria P, Jungers P. Encrusted pyelitis: early diagnosis facilitates conservative management. Presse Med. 37(1 Pt 2), 85-87 (2008).

22. Jost BH, Billington SJ. Arcanobacterium pyogenes: molecular pathogenesis of an animal opportunist. Antonie Van Leeuwenhoek 88(2), 87-102 (2005).

23. Levy CE, Pedro RJ, Von Nowakonski A, Holanda LM, Brocchi M, Ramos MC. Arcanobacterium pyogenes Sepsis in Farmer, Brazil. Emerg. Infect. Dis. 15(7), 1131-1132 (2009).

24. Plamondon M, Martinez G, Raynal L, Touchette M, Valiquette L. A fatal case of Arcanobacterium pyogenes endocarditis in a man with no identified animal contact: case report and review of the literature. Eur. J. Clin. Microbiol. Infect. Dis. 26(9), 663-666 (2007).

-• Shows that Arcanobacterium can rarely infect humans, causing fatal infections. Moreover, it proves that an animal contact is not essential for contamination. 
25. Chesdachai S, Larbcharoensub N, Chansoon T et al. Arcanobacterium pyogenes endocarditis: a case report and literature review. Southeast Asian J. Trop. Med. Public Health 45(1), 142-148 (2014).

-. Shows that Arcanobacterium can be a cause of fatal human infections.

26. Kavitha K, Latha R, Udayashankar C, Jayanthi K, Oudeacoumar P. Three cases of Arcanobacterium pyogenes-associated soft tissue infection. J. Med. Microbiol. 59(Pt 6), 736-739 (2010).

27. Jost BH, Songer JG, Billington SJ. Cloning, expression, and characterization of a neuraminidase gene from Arcanobacterium pyogenes. Infect. Immun. 69(7), 4430-4437 (2001). 